\title{
Location planning of distributed energy stations based on improved P-median model
}

\author{
Zhengji Meng ${ }^{1 *}$, Xiaoguang $\mathrm{Hao}^{1}$, Shiyan $\mathrm{Liu}^{1}$, Jianfeng $\mathrm{Li}^{1}$ \\ ${ }^{1}$ Electric Power Research Institute of State Grid Hebei Electric power Company, Shijiazhuang, Hebei, 050051, China
}

\begin{abstract}
The integrated energy system creates the possibility for the interconnection and coordination of different energy sources, and is an effective means to improve the energy use of the system, increase energy efficiency, and reduce environmental pollution. At present, the planning of distributed energy stations for integrated energy systems mostly focuses on equipment selection and equipment capacity. However, there are relatively few studies on the location of energy stations and pipeline layout planning. Firstly, this paper proposes a distributed energy station location method based on the improved p-median model, which combines the energy supply path of the energy station with the actual transportation network, and introduces the weight coefficient of multi energy load to reflect the diversity of energy demand of load. Finally, the specific solution method is given, and the rationality and feasibility of the proposed method are verified by an example.
\end{abstract}

\section{Introduction}

In recent years, with the rapid development of social economy, the demand for energy consumption is increasing. To alleviate the environmental problems caused by coal-based energy structure has become a difficult problem for many countries ${ }^{[1]}$.Unified coordination of different energy systems, building regional integrated energy system (RIES) has become the focus of many scholars ${ }^{[2]}$.Distributed energy station (DES) integrates multiple energy devices and is the core of RIES ${ }^{[3]}$.At present, the planning and Research on DES are mostly focused on the configuration plan of internal integrated equipment ${ }^{[4]}$. In fact, determining the location of DES and the energy transmission pipeline network path layout is also the key to ensure the economic benefits of the system.

Especially for regional integrated energy systems with multiple DES, the distance between DES and the layout of pipelines are closely related to the location of DES. Determining the construction location and supply load of each DES through reasonable planning will help to reduce the construction cost of the energy supply network and improve the economy of the regional comprehensive energy system.

Most of the existing studies do not consider the impact of regional energy conditions, types and municipal road planning on pipeline layout ${ }^{[5-6]}$. Therefore, based on the $\mathrm{p}$-median model $\mathrm{l}^{[7]}$, this paper studies a DES location method considering the actual construction distance of pipe network and the demand of load multi energy. This method combines the energy supply path of DES with the actual transportation network, and introduces the weight coefficient of multi energy load, which reflects the diversity of energy demand (electricity and heat). Combined with the load energy consumption information, the model can determine the construction location of multi regional energy station and the amount of multi energy load supplied by the energy station.

\section{Location planning of distributed energy stations based on improved P. median model}

\subsection{Basic principle of $p$ - median model}

In general, the location problem of energy station can be abstracted into a mathematical problem composed of "points" and "edges", and solved by means of graph theory. Among them, the energy station and energy users are the nodes of the network, and the pipelines or lines transporting energy are the edges of the network. It belongs to the p-median problem in the energy network to select one or $\mathrm{P}$ nodes from a number of nodes to be selected, and the energy stations on these nodes are connected with the users to realize energy supply. The advantage of p-median model is that most of the models are linear optimization models. Compared with nonlinear optimization, linear optimization model has many advantages in solving speed and accuracy.

Median point definition: The shortest distance between two nodes $n$ and $m$ in graph $S$ is represented by $d_{n m}$, which represents the sum of the distances from node i to all nodes in the graph, that is:

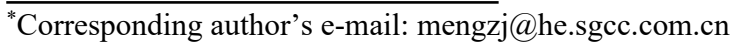




$$
A d_{n}=\sum_{n=1}^{N} d_{n m} \quad(i=1,2, \cdots, m)
$$

The node $n$ with the smallest distance is called the median point in graph $S$.

With the above definition, the problem of energy station location based on P-median can be summarized as: there are $n$ multi-energy load nodes and $m$ candidate location nodes in planar S. How to minimize the sum of authorized distances for energy users by calculating how to select $\mathrm{P}$ suitable nodes from $\mathrm{m}$ candidate locations. Taking the energy station location problem of $n=7, m=4$, $P=2$ as an example, the location process can be shown in Fig.1.

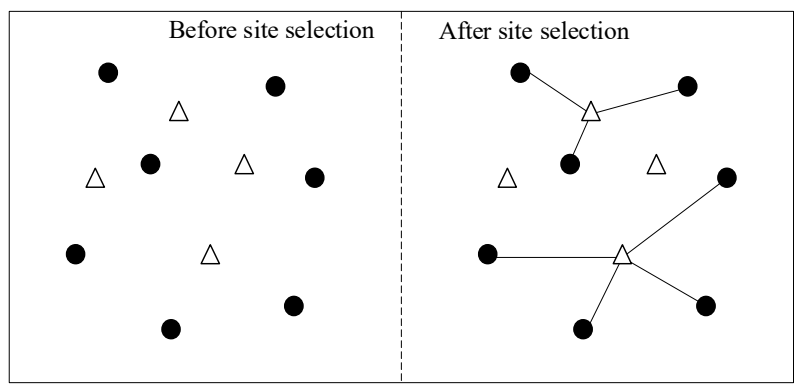

Load node $\triangle$ DES candidate position - Energy supply path

Figure 1. DES Location Problem Diagram $(n=7, m=4, P=2)$.

\subsection{Location planning of DES based on improved P-median model}

For regional comprehensive energy system planning and construction, in order to save urban underground space and reduce the impact of repeated excavation of pavement on environmental traffic during the construction of energy pipelines, engineering pipelines are usually laid out along the edge of traffic roads ${ }^{[8]}$. At the same time, the selection process of energy station site also needs to meet the local geographic environment, resource conditions, and municipal planning restrictions, and make a primary selection of each energy station location.

At the same time, the selection process of energy station site also needs to meet the local geographic environment, resource conditions, and municipal planning restrictions, and make a primary selection of each energy station location. Therefore, the selection of energy station sites and pipeline transmission routes should fully consider regional resource conditions and the status of municipal road network planning. Moreover, unlike single energy system planning, users in integrated energy systems have multiple forms of energy use, and site planning for energy stations also needs to take into account the diversity of energy needs of different users.

Based on the above analysis, this paper improves the basic P-median model. Firstly, it combines the energy supply path of the energy station with the actual traffic road, and takes the shortest distance along the road as the shortest distance of the energy supply path of the energy station. Its planning diagram is shown in Figure 2. Secondly, by introducing the multi-energy load weight factor, the diversity of load demand for energy (electricity, heat) is reflected.

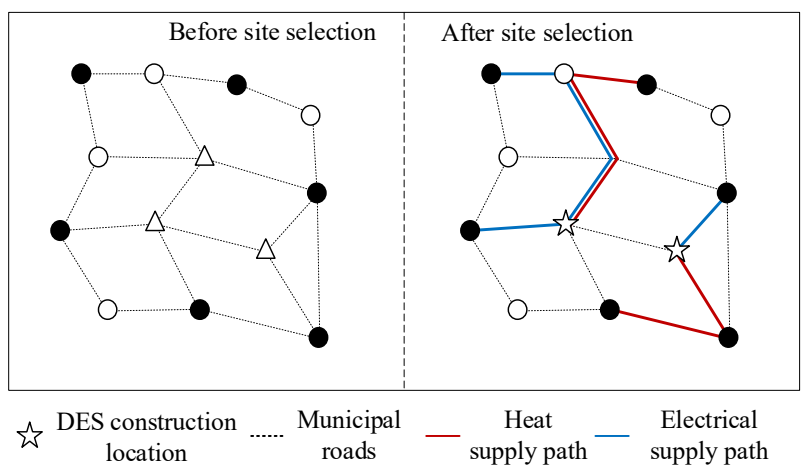

Figure 2. DES location planning based on road network ( $n=6$, $m=3, P=2)$.

On the basis of the above principles, this paper integrates multi-energy load node information, DES candidate location information, energy pipeline construction cost and other conditions, and establishes a model with the goal of the lowest total investment cost of DES energy supply pipeline network. The constructed model can be expressed as:

$$
\begin{aligned}
& \min \sum_{n \in \Gamma^{N}} \sum_{m \in \Gamma^{M}} \omega_{n} d_{n m} Y_{n m} \\
& \text { s.t. } \sum_{n \in \Gamma^{N}, m \in \Gamma^{M}} Y_{n m}=1 \\
& \sum_{m \in \Gamma^{M}} X_{m}=\mathrm{P} \\
& Y_{n m} \leq X_{m} \\
& X_{m}, Y_{n m} \in\{0,1\} \quad \forall n \in \Gamma^{N}, \forall m \in \Gamma^{M}
\end{aligned}
$$

Where $\Gamma^{N}$ is a collection of nodes with multi-energy load, $\Gamma^{M}$ is a collection of positions to be built for DES; $\omega_{n}$ is the multi-energy load weight coefficient, it represents the weight of load node $n$ supplying energy through energy station $m, d_{n m}$ is representing the shortest distance between load node $\mathrm{n}$ and each energy station $\mathrm{m}$ along the municipal road; $X_{m}$ indicates whether the position to be selected is selected, if node $\mathrm{m}$ is selected to build an energy station, it will be 1; otherwise, it will be $0 ; Y_{n m}$ indicates whether the load node $\mathrm{n}$ supplies energy through the energy station $\mathrm{M}$. if so, it is 1 ; otherwise, it is 0 .

The above multi energy load weight coefficient $\omega_{n}$ specifically represents the unit cost required by load node $n$ to construct the pipeline.

$$
\omega_{n}=\lambda_{n}^{\mathrm{e}} C_{n}^{\text {line }}+\lambda_{n}^{\mathrm{h}} C_{n}^{\mathrm{pipe}} \quad \forall n \in \Gamma^{N}
$$

where $\lambda_{n}^{\mathrm{e}}$ and $\lambda_{n}^{\mathrm{h}}$ indicate whether the load node $n$ needs power or heat supply. If so, it is 1 ; otherwise, it is 0 ; $C_{n}^{\text {line }}$ and $C_{n}^{\text {pipe }}$ indicate the unit construction cost of power lines and heating pipelines corresponding to load 
node $n$. The relationship between unit length and rated capacity can be obtained by fitting:

$$
\left\{\begin{array}{l}
C_{n}^{\text {line }}=0.0956 P_{n_{-} \max }^{\mathrm{e}}-77.7785 \\
C_{n}^{\text {pipe }}=0.0220 P_{n_{-} \max }^{\mathrm{h}}+21.0228
\end{array} \quad \forall n \in \Gamma^{N}\right.
$$

In order to meet the energy supply of the energy station to the load node, $P_{n_{-} \max }^{\mathrm{e}}$ and $P_{n_{-} \max }^{\mathrm{h}}$ indicate the maximum electric power and thermal power required by the load node $n$.

\subsection{The solution for planning model}

The shortest distance of the energy supply pipeline in the above-mentioned improved P-median model is the shortest distance between the load and each energy station along the municipal road. In this paper, Dijkstra shortest path solving algorithm ${ }^{[9]}$ is used to solve the shortest distance in the above grid. The basic solution process is shown in Figure3:

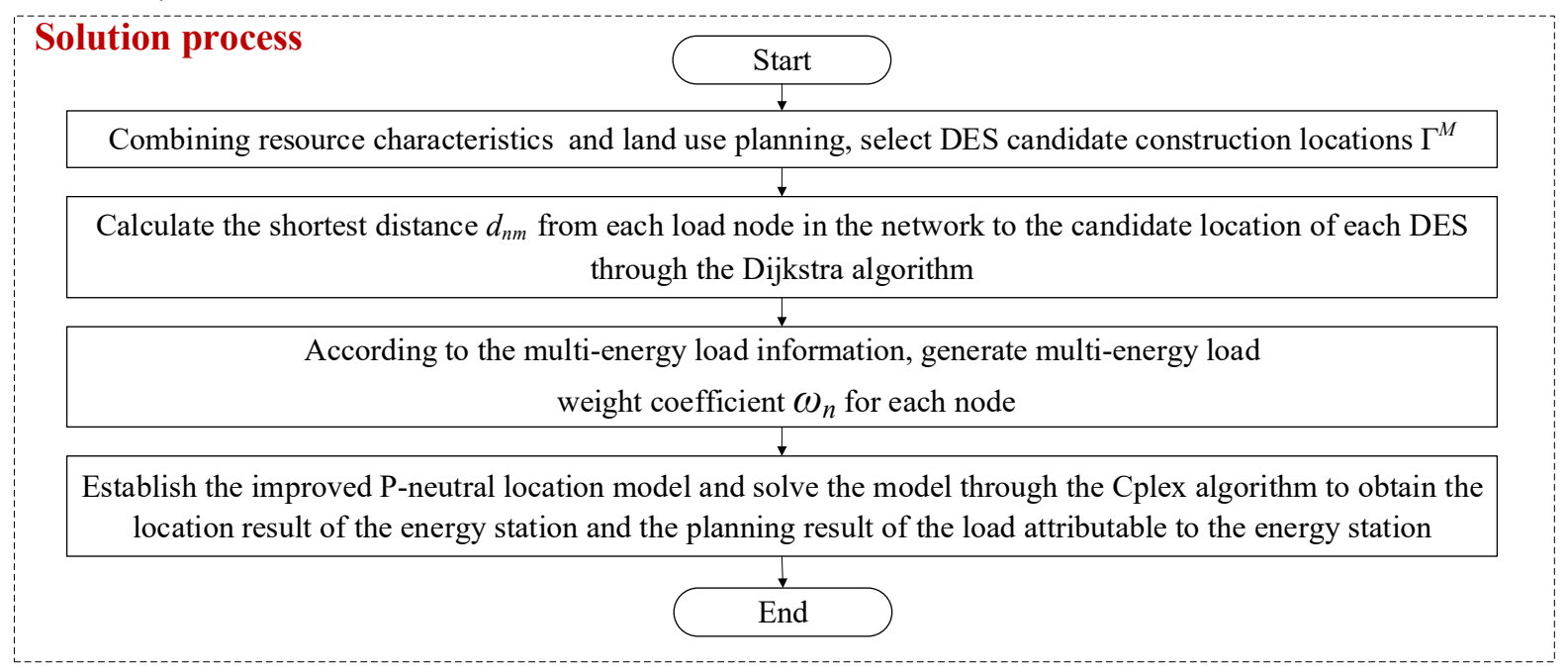

Figure 3. Flow chart of solving planning model.

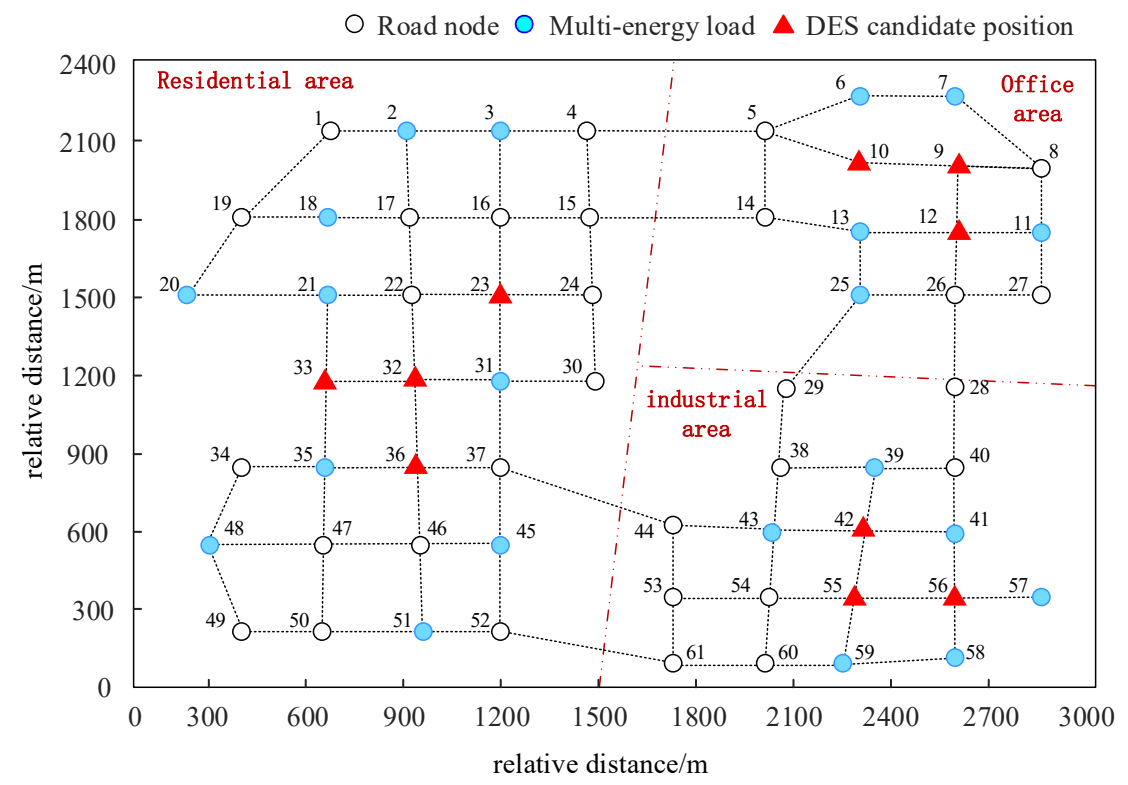

Figure 4. Flow chart of solving planning model.

\section{Case studies}

\subsection{Case description}

Taking an area in the north as an example, the area can be roughly divided into three areas: residential area, office area and industrial area. The traffic network layout and multi energy load distribution of each area are shown in Figure 4. The energy demand of each load node is shown in Figure 5. In this paper, three energy stations are planned to meet the energy demand of power and heat for each region load. The candidate locations of energy stations obtained by combining the local resource characteristics and land planning properties are shown in Figure 4. 

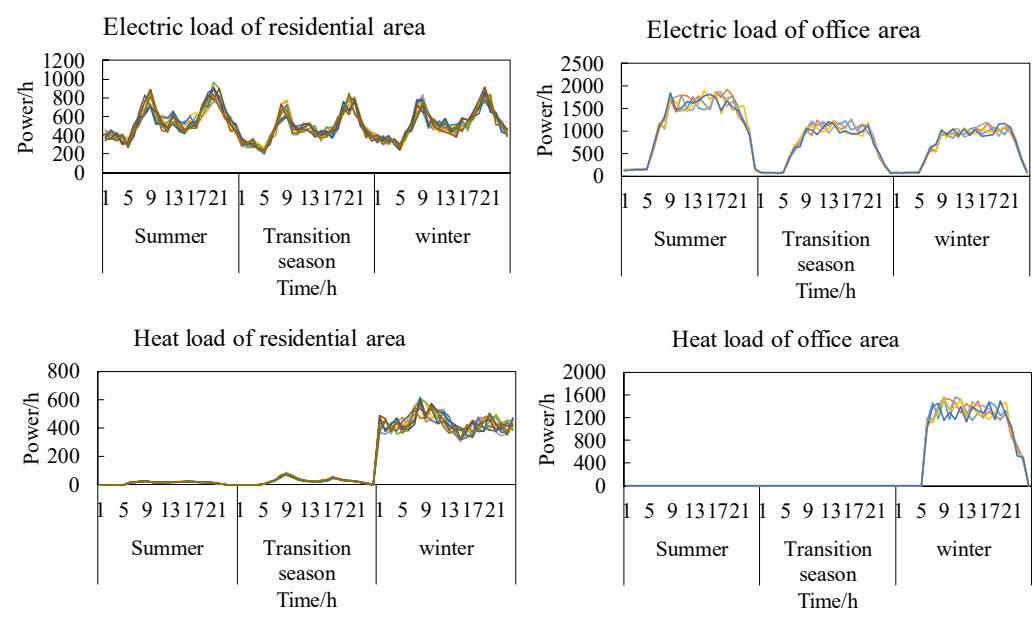
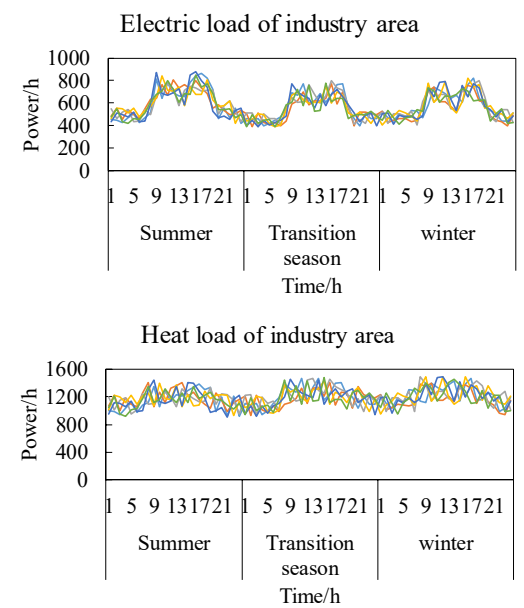

Figure 5. Load curve of each node in each area (electricity and heat).

\subsection{Case analysis}

the results are shown in Figure 6. Among them, energy stations 1,2 , and 3 provide energy supply to residential

Based on the load characteristic curve of each region, the location of the three DES in the region was planned, and

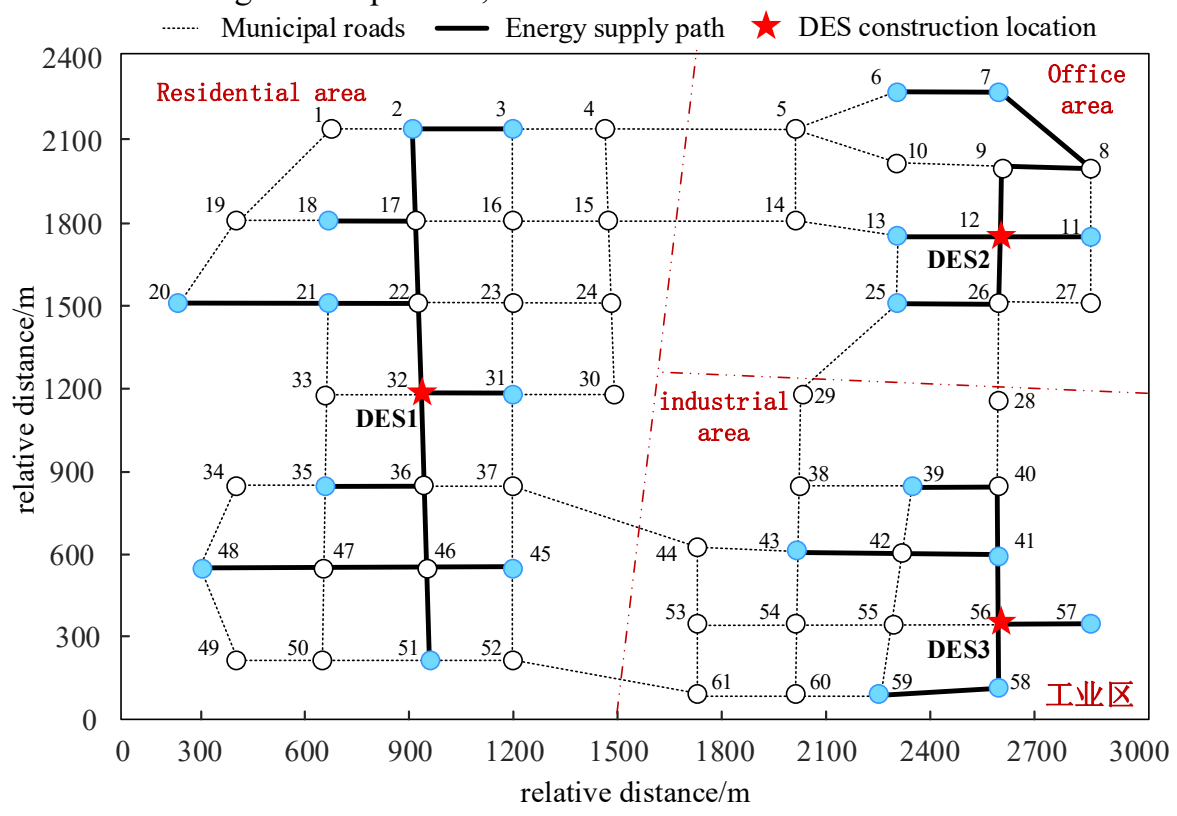

Figure 6. Energy station location planning results.

\section{Conclusions}

This paper studies a DES location planning method based on the improved P-median model, which combines the energy supply path of the energy station with the actual transportation network, and introduces the multienergy load weight coefficient to reflect the diversity of load for energy demand (electro-thermal). Combining the load energy information to solve the model, the construction location of each area DES and the layout of the energy supply network can be determined.

\section{Acknowledgments}

I am very grateful to my mentor, Dr.Xiaoguang Hao, for his careful guidance of my graduation thesis in the past six months, which greatly improved my understanding of academic writing and taught me a lot of specific research skills.

\section{References}

1. Zeng Ming. Key supporting technologies for the future development of distributed energy in the background of energy internet[J]. The Age of Electricity, 2018(1): 36-37. 
2. Tan Zhukui, Cheng Yuefeng, Shi Shouyuan, et al. Discussion on key technologies of energy internet access equipment $[\mathrm{J}]$. Power System Protection and Control, 2019, 47(14): 18-19.

3. Bai Xuexiang, Zeng Ming, Li Yuanfei, et al. The model and algorithm of thermoelectric collaborative planning of regional energy supply network[J]. Power System Protection and Control, 2017, 45(5): 65-72.

4. Sheikhi A, Rayati M, Ranjbar A M. Energy hub optimal sizing in the smart grid; machine learning approach[C]//Innovative Smart Grid Technologies Conference. Denver: IEEE, 2015: 1-5.

5. Maroufmashat A, Elkamel A, Fowler $\mathrm{M}$, et al. Modeling and optimization of a network of energy hubs to improve economic and emission considerations[J]. Energy, 2015(93): 2546-2558.

6. Maroufmashat A, Sattari S, Roshandel R, et al. Multi-objective optimization for design and operation of distributed energy systems through the multi-energy hub network approach[J]. Industrial \& Engineering Chemistry Research, 2016, 55(33): 8950-8966.

7. Long Weiding, Bai wei, et al. Community energy planning for built environment in low carbon cities [M]. China Architecture \& Building Press, China Architecture \& Building Press.

8. Wang Zhaoqiang, Liu Qingrong, Ruan Yingjun, et al. Research and application of optimal layout method for station network layout in regional energy planning $[\mathrm{J}]$. Journal of Engineering for Thermal Energy and Power, 2019(8): 25-30.

9. Zhang Fuhao, Liu Jiping, Li Qingyuan, et al. A New Way of Network Analysis Based on Dijkstra[J]. Journal of Harbin University of Science and Technology, 13(3), 35-37. 